\title{
REDUCTION IN THE PILOT'S STRESS DURING THE LANDING MANEUVER BY PROVIDING ACCURATE HEIGHT INFORMATION
}

\author{
Miroslav Šplíchal $^{1}$ \\ ${ }^{1}$ Institute of Aerospace Engineering \\ Brno University of Technology \\ Technická 2, 61669 Brno, Czech Republic \\ splichal.m@fme.vutbr.cz \\ ORCID: 0000-0002-3223-9142
}

Keywords: Landing, Stress measurement, Heart rate variability

\begin{abstract}
The article is focused on pilot stress reduction during landing by providing accurate height information. It is based on the results of the completed project TA04030510 - Assistance systems supporting the pilot's right decisions increasing the safety of operating small civil aircraft. The landing is the most critical phase of a flight. Accidents occurring during approach and landing phases generally represent up to $40 \%$ of all aircraft accidents. A frequent cause of these accidents is a pilot error. During project development, 112 accidents of ultralight aircraft and 152 accidents of small GA aircraft that happened from 2005 to 2015 were analyzed. In total, 48 aircraft landing-related accidents and 22 accidents in ultralight aircraft were found. Landing accidents represent $26.5 \%$ of all investigated accidents.
\end{abstract}

As a solution to the typical causes of accidents (for instance inability to set the flare altitude correctly etc.), a high-precision indication system was developed within the project. The system is aimed at reduction of pilot workload and thus decrease of height related estimation errors. After the initial flight verification, the system was subjectively evaluated by pilots. The results showed different changes in the perceived pilots stress. Pilots subjectively rated the system worse than expected. Subjective assessments may distort personal attitudes, so another experiment was performed. Monitoring of heart rate variability (HRV) was selected as an effective and practical indicator of pilot stress intensity and as an objective indicator.

Commonly available equipment and procedures were used for monitoring of HRV. From a variety of analytical approaches, the Poincare method was subsequently adopted as the most practical and easily applicable. The results indicate that it is possible to measure the HRV in a real flight environment and the results found correspond with the conclusions of other studies.

\section{INTRODUCTION}

Landing is the most challenging phase of a flight. The aircraft is moving at low altitude and low speed, which limits the possibilities of manoeuvring. The pilot should maintain a trajectory in a narrow approaching corridor that will ensure a successful landing. In a small plane and on a grass strip, the pilot is dependent only on his abilities and senses. Accidents occurring in the approach and landing phases generally represent up to $40 \%$ of all aircraft accidents. A similar proportion applies to the 
general aviation category where, according to the NTSB statistics [17], the landing related accidents account for approximately one third of all aviation accidents. A number of projects were targeting at this high accident rate in the past. The development in the field of electronics and the reduction in the price of MEMS sensors allows designing advanced assistive systems that may have the potential to prevent some accidents. The project TA04030510 - "Assistance systems supporting the pilot's right decisions increasing the safety of operating small civil aircraft" focused on the development of such assistance systems. The author has actively participated in this project. The project TA04030510 investigated 112 accidents of ultralight aircraft and 152 accidents of GA aircraft that happened from 2005 to 2015 in the Czech Republic. In total, 48 general aviation aircraft landing-related accidents and 22 accidents of ultralight aircraft, respectively, were found. It represents $31.5 \%$ for the GA category up to 5700 MTOW and $19.6 \%$ for ultralight aerodynamically controlled aircraft. In addition, the proportion of these accidents is relatively constant over the course of time, indicating the same cause - a pilot error. Within the investigated 70 landing accidents, 98 pilot errors were identified. The greater number of identified pilot errors is due to a combination of different types of errors in one accident. The error classification was selected according to the Reason human error model. The cause of pilot errors is not unknown: it is the pilot's stress and workload. Stress is typically associated with excessively high workload levels. The relationship between stress and performance has been verified in many experiments and the Yerkes-Dodson Law is often mentioned: the relationship between stress and performance has an inverted U-shape. The law indicates that the performance increases with physiological or mental stress, but only up to a point. Beyond this point, any further increase in the stress level reduces the power and the human is prone to errors. To improve the performance, the workload or stress should be reduced. Nowadays the small-aircraft pilot is dependent only on his abilities. A lack of self-confidence in these abilities can lead to a higher level of pilot's stress. As a result, a loss of situational awareness may occur, and the pilot may make mistakes that lead to an accident.

Although each accident is unique, it is possible to identify some common signs or scenarios of accidents. Accident investigation in the project has shown that in landing accidents were prevailing skill-based errors and perceptual errors. These error categories can be associated with the pilot's stress. As a possible solution, a flare altitude signalling system was designed. From a psychological point of view, such a system can reduce the pilot's stress from a challenging task. In the system design the findings from the Benbassat experiment in 2002 [1] were used. The Benbassats experiment demonstrated the effect of using acoustic information to improve the performance of the pilot during the landing maneuver. Original experiments were performed only on a simulator in laboratory conditions. Our research was followed by the mentioned Benbassat experiment, when the height signalling system was designed and manufactured. The main idea was to reduce the pilot error caused by an incorrect estimation of height. The system should also reduce the pilot's stress from the landing maneuver because the pilot can feel better control and higher situational awareness.

\section{SYSTEM CONCEPTION AND DEVELOPMENT}

The developed device consisted from a laser altimeter unit SF02, which provided continuous information about the height above ground up to 30 meters and a computing unit which performed signal filtration for sudden changes in height and for generating an acoustic signal in the range of $4 \div$ $2 \mathrm{kHz}$. The system was also supplemented with visual signaling via the LED panel. More details about the system are given in [13]. The basic components of the system and their deployment on the aircraft are shown in Figure 1. 
Acoustic signaling is only active up to the height of 1 meter above the runway. The device was tested in the real environment on the ultralight airplane type Zenair $\mathrm{CH} 601$ because the flight and landing of a small aircraft is too difficult to simulate. The system was initially tested by five pilots of different ages and with different flight experiences. A limited set of pilots was given by the method chosen. The pilots participating in the experiment had to be able to control the aircraft.

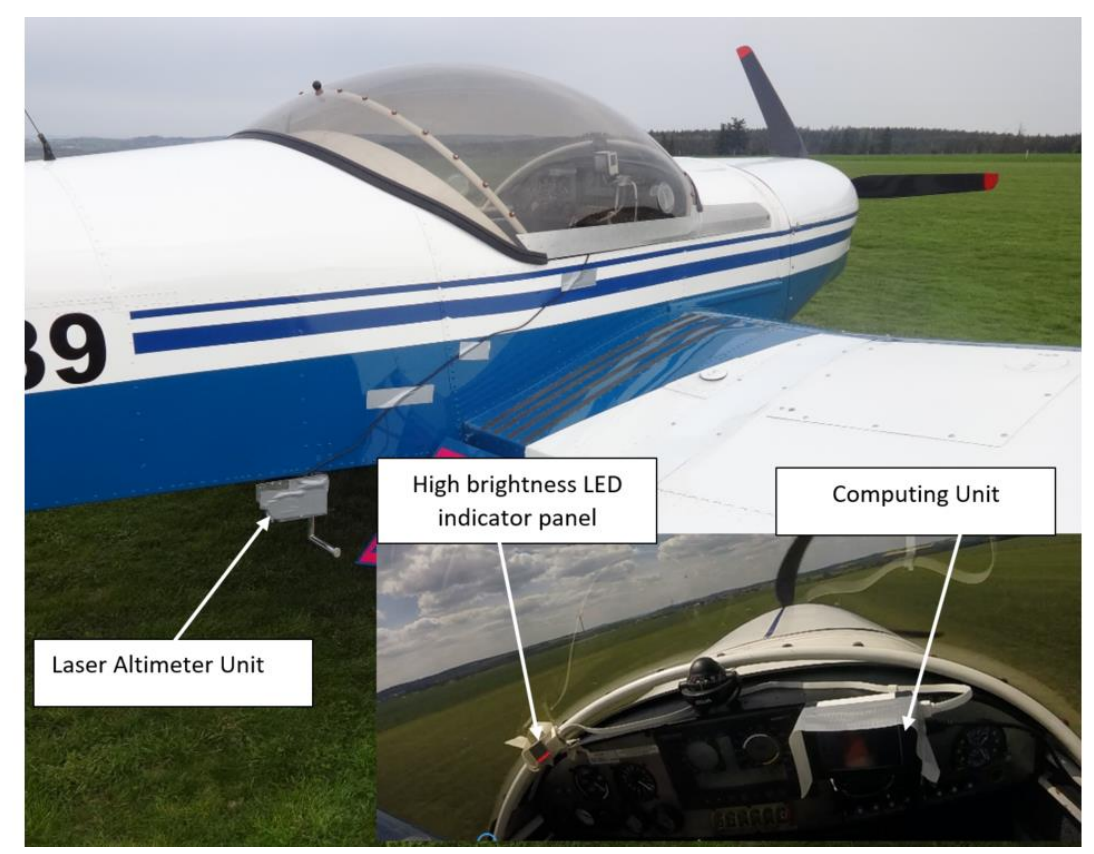

Figure 1: System installed on the Zenair CH601 airplane

The pilots were supposed to assess the benefits of the system in terms of the reduced perceived taskload during landing. The NASA-TLX method was used to measure the load. Each pilot completed a questionnaire immediately after finishing the flight, which investigated the six following factors: mental difficulty, physical difficulty, time intensity, performance, effort and level of frustration. Each of these factors was expressed on a 20-point scale labeled Low / High or Good / Bad. In addition, significance was assigned to each category. Based on these values, the overall score was determined according to the method given in [8], which corresponded to the perceived workload. The questionnaires were processed with a modified Python script originally created by Jonathan Polom available on the Github. The results are summarized in the graph in Figure 2. 


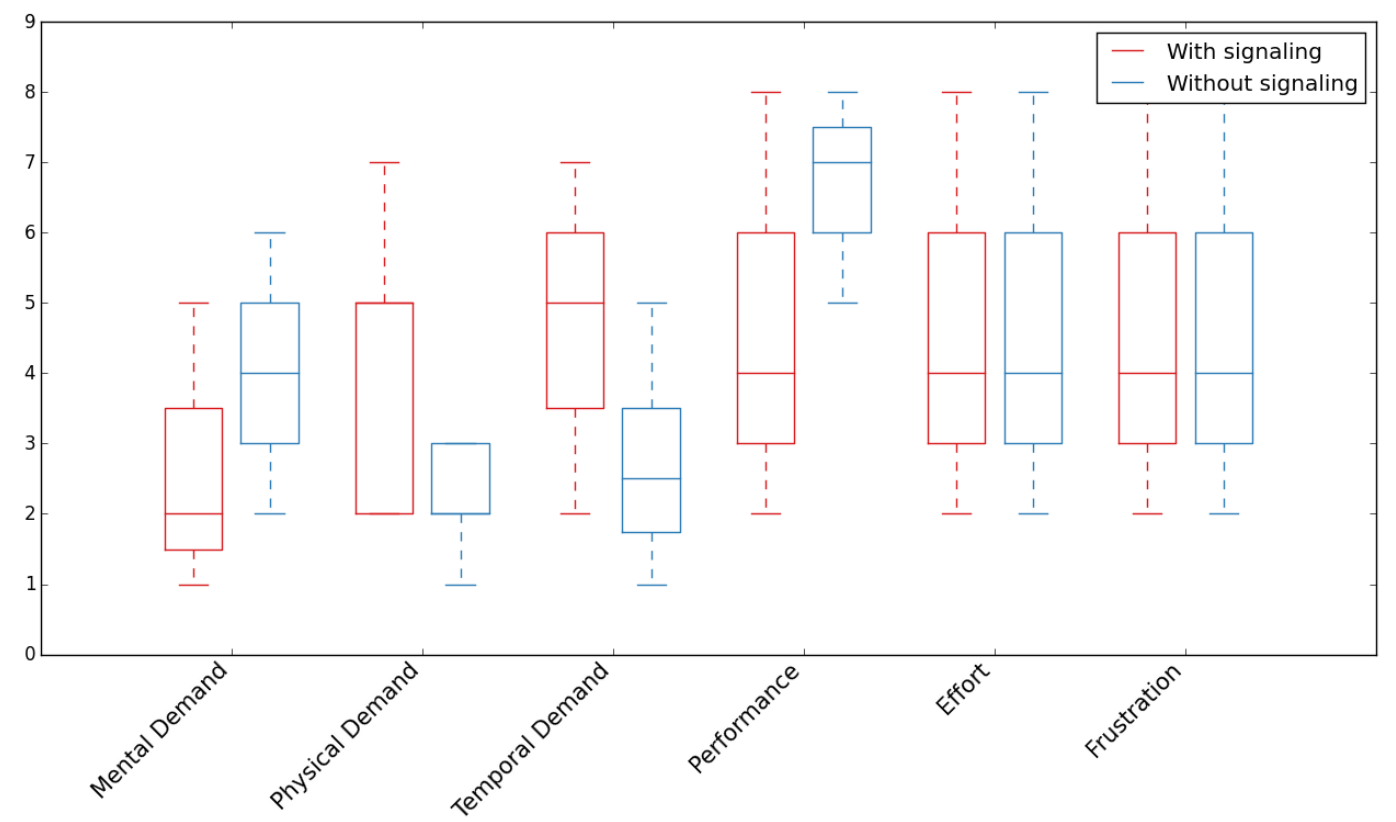

Figure 2: NASA-TLX Pilots workload

The results show that there was a reduced load during the landing with the total score of 55 points for height signaling versus 57 points for landing without signaling. The perceived workload of the pilots decreased, but this drop was lower than expected, only by two points. In particular, the results found in the Effort and Frustration categories are inconsistent with the expectations. Pilot's frustration was higher for landing with signaling. The evaluation results are probably related to the higher expectations of pilots. The effort category does not show a significant reduction for landing with signaling.

In addition, the pilots were supposed to express their own subjective opinion on the change in the level of stress during landing with signaling versus landing without signaling. Likert's rating scale was selected. Pilots could choose the rating of five grades 1 - low, 2 slightly lower, 3 - no change, 4 slightly higher, 5 increased. The evaluation results are shown in Figure 3.

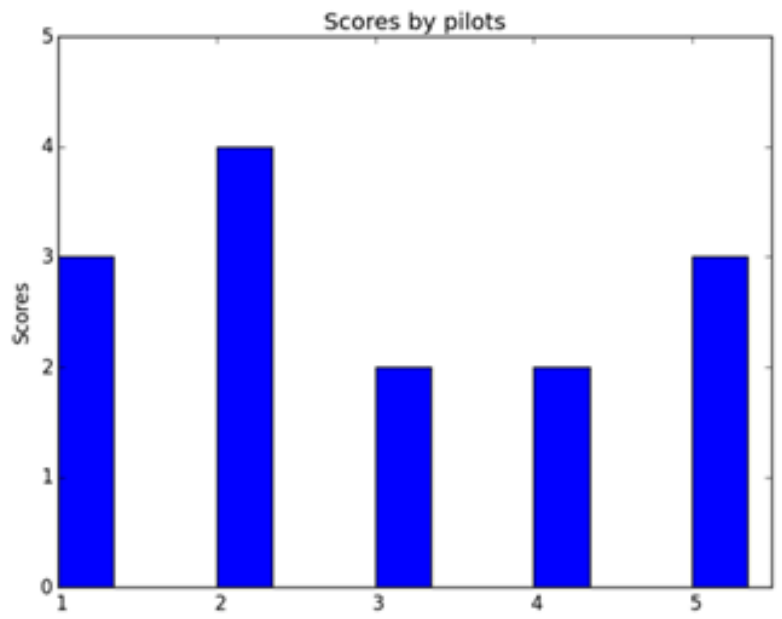

Figure 3: Relative change in stress level evaluated by pilots for landing with signaling 
The overall record score was 2.8, thus the pilot's stress was reduced. Experienced pilots reported that there was no change in the level of perceived stress. Less experienced pilots then reported a slight decrease in perceived stress. One pilot reported an increase in stress because he felt a contradiction between signaling and his feelings.

Both methods used to evaluate the system are very subjective. Also such a limited number of involved pilots is not sufficient to produce any demonstrable results. For this reason, there was a need for an objective verification of the system.

\section{MEASUREMENTS OF STRESS}

It is known that stress influences the balance of Autonomous Nervous System. For this reason, several studies investigated the cardiovascular reaction induced by stress using the Heart Rate Variability as an easily measurable physiological parameter. The heart rate variability (HRV) consists of changes in the time intervals between consecutive heartbeats called interbeat intervals (IBIs). The oscillations of a healthy heart are complex and constantly changing, which allows the cardiovascular system to adjust rapidly to sudden physical and psychological challenges to homeostasis. The HRV signal contains well-defined rhythms, which have been successfully shown to contain psychophysiological information [3]. The HRV application in aviation has been dealt with in several studies $[4,5,6]$. Veltman and Gaillard [16] used pilots in a flight simulator with a flight scenario with 4 levels of maneuvering/pursuit difficulty. Tattersall and Hockey [15] examined flight engineers in a flight simulator using HR and the mid- and high-bands of the HRV spectrum. These experiments were conducted on flight simulators. The benefit of our research is to verify the use of HRVs to assess the level of stress in a real flight environment. The review conducted found no record about a similar experiment. The heart rate indicates only relative differences in workload. Moreover, each individual has a slightly different value of the HRV, which can be influenced by many factors. For this reason, it is necessary to create a baseline for comparison. Different levels of stress have been assessed in our experiment. As the baseline, a horizontal flight and a relaxed state when the person was 10 minutes at rest were selected.

\section{THE EXPERIMENT}

The HRV is generally obtained by monitoring the peak intervals ( $R-R$ intervals) in an electrocardiogram (ECG). The heart rhythm must be continuously monitored by a suitable device. $A$ standard medical device designed to measure the ECG type CARDIAX was tested as the default device. This device proved to be inappropriate because it contains pre-processing and filtering of the measured signal while for a successful measurement the subject must be at rest. Other solutions like chest straps for fitness activities were not appropriate because they have a closed system that does not allow HRV to be analyzed. As an alternative, a simple AD8232 module was selected which is able to measure raw data. This module, in conjunction with the STM32 microcontroller, was used to record data. The accuracy and suitability of this device was verified by a test measurement where, under the same conditions, a comparable output of both the medical device and the selected device was verified. The ECGs electrode was of key importance for a successful measurement. There was a problem with the safety belts in the airplane that affected the ECGs electrodes. After several experiments with different electrodes it was necessary to use a suction ball electrode. These electrodes are uncomfortable and unsuitable for long-term application but provide the best results.

Two pilots participated in the experiment, further referred to as Pilot 1 and Pilot 2. Pilot 1 had about 8 fly hours on the airplane and pilot 2 had over 100 fly hours on the airplane. Each pilot made two flights. The first flight was without altitude signaling, the second flight was with signaling altitude 
above the terrain. In addition, a control measurement was carried out where the pilots were at rest and relaxed, which serves as a reference. Recordings of 3-minute segments capturing flying on the horizon and during landing were isolated from the ECG records. In these isolated data segments R-R intervals were manually identified and further analyzed.

\subsection{The method of HRV data analysis}

The methods of HRV analysis can be divided into two groups: linear and non-linear methods. Linear methods can be used in time or frequency domain for the HRV analysis [2]. Time-domain parameters are statistical calculations of consecutive RR time intervals and they are correlated with each other. Frequency-domain parameters are based on spectral analysis. The linear time and frequency domain techniques for HRV were standardized in a report of the Task Force of the European Society of Cardiology and the North American Society of Pacinian Electrophysiology [14]. Recently, the interest for non-linear analysis has been constantly increasing as the HRV measurements are non-linear and non-stationary and a considerable part of information is coded in the dynamics of their fluctuation in different time periods. At present, there are many non-linear methods. For the purpose of our study, a simple graphical nonlinear method called Poincaré plot $[7,9]$ was chosen. The method provides summary information as well as detailed beat-to-beat information on the behavior of the heart. It is a graphical representation of temporal correlations within the RR intervals derived from ECG signal. The Poincaré plot is known as a return maps or scatter plot, where each RR interval from the time series $R R=\{R R 1, R R 2, \ldots, R R n, R R n+1\}$ is plotted against the next $R R$ interval. A quantitative analysis of the HRV attractor displayed by the Poincaré plot can be made by adjusting it to an ellipse. For the performance analysis, the SD1 (Standard Deviation1), SD2 (Standard Deviation 2) were used as evaluation parameters [9]. A software tool Python Pylab was used for the calculation of SD1 and SD2 parameters from the IBI series. SD1 and SD2 were determined according to the methodology given in [7]. These indexes are measurements of the heart variability over a single beat [21]. Index SD1 represents a measurement of short-term heart rate variability caused by respiratory sinus arrhythmia and index SD2 represents a measurement of a long-term heart variability. Physiologically, SD1 is thought to be mediated by the vagal parasympathetic activity whereas SD2 is influenced by both parasympathetic and sympathetic activity [3]. After reviewing a series of papers related to mental stress and HRV, the SD1/SD2 ratio derived from Poincaré plot was used as an indicator of mental stress. Examples of the Poincaré plot of pilot 1 for different phases of the flight are in shown the graph in Fig.4.
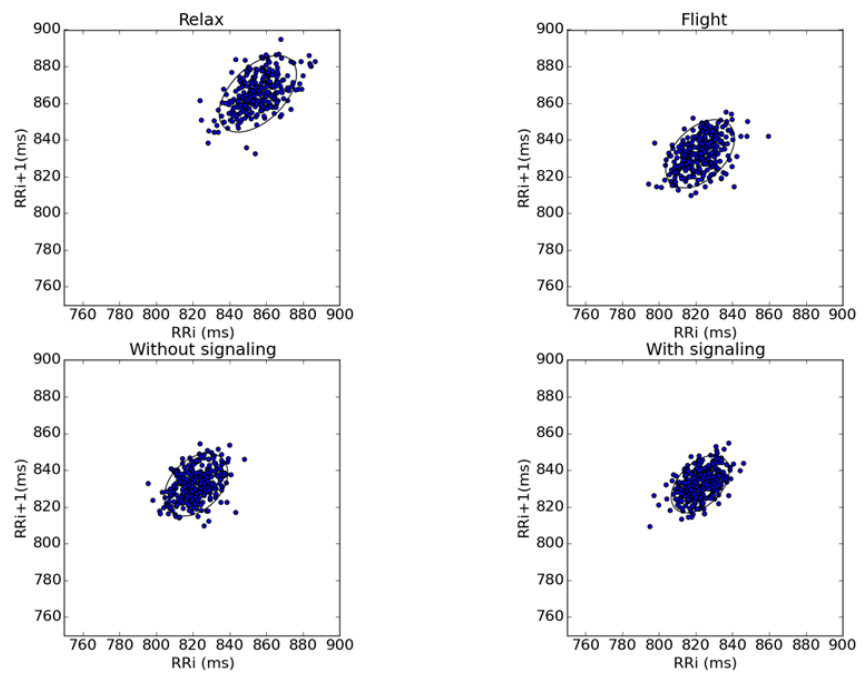
Figure 4: Poincaré plot of Pilot 1 for different flight phases with fitted ellipses

\begin{tabular}{|c|c|c|c|c|c|c|}
\hline Activity & $\begin{array}{c}\text { SD1 } \\
\text { Pilot } 1 \\
\end{array}$ & $\begin{array}{c}\text { SD2 } \\
\text { Pilot } 1 \\
\end{array}$ & $\begin{array}{l}\text { SD1/SD2 } \\
\text { Pilot1 } \\
\end{array}$ & $\begin{array}{c}\text { SD1 } \\
\text { Pilot } 2 \\
\end{array}$ & $\begin{array}{c}\text { SD2 } \\
\text { Pilot } 2 \\
\end{array}$ & $\begin{array}{c}\text { SD1/SD2 } \\
\text { Pilot2 }\end{array}$ \\
\hline Relax & 55.20 & 110.01 & 0.501 & 55.78 & 102.01 & 0.546 \\
\hline Flight & 34.92 & 74.12 & 0.471 & 31.39 & 65.78 & 0,477 \\
\hline Lading with signal & 28.14 & 65.78 & 0.428 & 23.73 & 66.14 & 0.359 \\
\hline $\begin{array}{l}\text { Landing without } \\
\text { signal }\end{array}$ & 27.68 & 66.34 & 0.417 & 24.02 & 67.67 & 0.355 \\
\hline
\end{tabular}

Table 1: SD1, SD2 and SD/SD2 ratio for Pilot 1 and Pilot 2 in different flight phases

\section{RESULT DISCUSSION}

The experiment results in table 1 show that both pilots had a change in their HRV. It is also obvious that the SD1/SD2 ratio varied with the load during the flight for both pilots. The lower SD1 /SD2 ratio can be associated with higher stress load. The SD1/SD2 ratio for landing is lower for both pilots than in case of the horizontal flight. This is consistent with the assumption that landing is more stressful than a horizontal flight. Concerning landing without signaling and with signaling, there is a greater difference in the SD1/SD2 ratio of the less experienced pilot. For both pilots, the SD1 / SD2 ratio, which can be associated with the stress level, did not increase when landing with signaling. For Pilot 1 , this ratio increased from 0.417 to 0.428 . The pilot stress may be lower when the landing height signalization is used. For Pilot 2 the ratio increased less from 0.355 to 0.359 this can correspond to his greater experience. The system could therefore reduce the level of stress of this pilot.

The initial results are consistent with the findings in the reviewed studies. Unfortunately, the number of pilots participating in the experiment is too small for a statistical verification of the theory. The main purpose of this experiment was to verify whether it is possible to realize the measurement in real conditions. It was also necessary to create and verify data processing procedures. These two main objectives have been achieved. The results indicate that the HRV can be used to assess the pilot's load even in a real environment.

\section{FUTURE WORK}

Experience gained in the experiment indicates that a reliable method of cardiac recording will be needed. ECG electrodes are of great importance for recording quality. The application of the selected electrodes is uncomfortable for the persons participating in the test, thus the use of the Polysomnography (PSG) is considered for a further development. PSG offers a very easy application, but also a number of problems with data analysis. Overcoming these difficulties would open up the ability to monitor the pilot's stress easily. It would also be possible to design new technologies in the human-machine interface.

\section{ACKNOWLEDGMENTS}

The research leading to these results has received funding from the MEYS under the National Sustainability Programme I (Project LO1202). 


\section{REFERENCES}

[1] Benbassat, D., Abramson C.I., (2002). Comparative Approach to Pilot Error and Effective Landing Flare Instruction. International Journal of Comparative Psychology, 15, 249-255

[2] Camm, A. J., et al., (1996).Heart rate variability: standards of measurement, physiological interpretation and clinical use., Task Force of the European Society of Cardiology and the North American Society of Pacing and Electrophysiology", Circulation, vol.93, no.5, pp. 1043-1065.

[3] De Vito, G., Galloway, S. D. R., Nimmo, M. A., Maas, P., \& McMurray, J. J. (2002). Effects of central sympathetic inhibition on heart rate variability during steady-state exercise in healthy humans. Clinical Physiology and Functional Imaging ,22(1), 32-38.

[4] Lee, Kyongsun,(2010). Effects of Flight Factors On Pilot Performance, Workload, And Stress At Final Approach To Landing Phase Of Flight, Electronic Theses and Dissertations. 1628. http://stars.library.ucf.edu/etd/1628

[5] Lee, Y.H., \& Liu, B.S. (2003). Inflight workload assessment: Comparison of subjective and physiological measurements. Aviation, Space, and Environmental Medicine, 74, 1078-1084.

[6] Li G, Baker SP, Grabowski JG, Rebok GW. (2001). Factors Associated with Pilot Error in Aviation Crashes. Aviation, Space and Environmental Medicine.

[7] Maud P.J., Fostrer C. (2006). Physiological Assessment of Human Fitness 2nd. Ed. ISBN:0-73604633-X

[8] NASA (2014). Task Load Index paper and pencil version. NASA Ames Research Center, Moffett Field, CA. http://humansystems.arc.nasa.gov/groups/TLX/downloads/TLXScale.pdf

[9] Piskorski J., P. Guzik,(2007). Geometry of the Poincare plot of RR intervals and its asymmetry in healthy adults", Physiological measurement, vol.28, n.3, pp.287-300.

[10] Roman J. et all. (1967). Flight research program VI: Heart rate and landing error in restricted fields of vision landings. Aerospace Med 38, 128-132

[11] Roscoe A.H. (1975). Heart rate monitoring of pilots during steep gradient, Aviat Space Environ 4ed 46, 1410-1415

[12] Ruffell Smith H.P. (1967): Heart rate of pilots flying aircraft on scheduled airline routes, Aerospace Med 38, 1117-1119

[13] Šplíchal M. (2017), Pilot errors during landing manoeuvres and possible ways to reduce them, New Trends in Civil Aviation: Proceedings of the 19th International Conference on New Trends in Civil Aviation 2017, p. 129-133, ISBN: 978-0-8153-7602-6

[14] Task Force of the European Society of Cardiology and the North American Society of Pacing and Electrophysiology. (1996). Heart rate variability: standards of measurement, physiological interpretation and clinical use. Circulation,93:1043-1065.

[15] Tattersall, A. J., \& Hockey, G. R. (1995). Level of operator control and changes in heart rate variability during simulated flight maintenance. Human Factors, 37, 682-698.

[16] Veltman J.A., Gaillard A.W.K, (1998). Physiological Workload Reaction to Increasing Levels of Task Difficulty, Ergonomics 41. 656-669

[17] Weener. E.F. (2015) General Aviation and the NTSB Most Wanted List, NTSB, April 25 


\section{COPYRIGHT STATEMENT}

The authors confirm that they, and/or their company or organization, hold copyright on all of the original material included in this paper. The authors also confirm that they have obtained permission, from the copyright holder of any third party material included in this paper, to publish it as part of their paper. The authors confirm that they give permission, or have obtained permission from the copyright holder of this paper, for the publication and distribution of this paper as part of the READ 2018 proceedings.

This is an open access article distributed under the Creative Commons Attribution License which permits unrestricted use, distribution, and reproduction in any medium, provided the original work is properly cited. (CC BY 4.0). 